\title{
ON THE STABILITY \\ OF VARIABLE STEPSIZE RATIONAL APPROXIMATIONS \\ OF HOLOMORPHIC SEMIGROUPS
}

\author{
C. PALENCIA
}

\begin{abstract}
We consider variable stepsize time approximations of holomorphic semigroups on general Banach spaces. For strongly $\mathrm{A}(\theta)$-acceptable rational functions a general stability theorem is proved, which does not impose any constraint on the ratios between stepsizes.
\end{abstract}

\section{INTRODUCTION}

Let $X$ be a complex Banach space, and let $A: D(A) \subset X \rightarrow X$ be the infinitesimal generator of a holomorphic semigroup $e^{t A}, t \geq 0$, of linear and bounded operators in $X$ (see, e.g., [15]). In this paper we are concerned with the problem of the stability of variable stepsize rational time approximations to the initial value problem

$$
\left\{\begin{array}{l}
u^{\prime}(t)=A u(t), \\
u(0)=u_{0}
\end{array}\right.
$$

Let us recall that the evolution operators associated with parabolic problems define holomorphic semigroups. Thus, holomorphic semigroups appear in many important problems. As is well known, a rational method for (1) replaces the operator $e^{t A}, t=k \Delta t, k \geq 1$ integer, by an operator $r(\Delta t A)^{k}$, where $r(z)$ is a rational approximation to the exponential. Basic examples include the backward Euler method, which is based on the rational function $r(z)=(1-z)^{-1}$, and the Crank-Nicolson method, which is based on the rational function $r(z)=$ $(1+z / 2)(1-z / 2)^{-1}$. The Lax stability of the method requires the boundedness for each $t>0$ of the powers $r(\Delta t A)^{k}, \Delta t=t / k, k>1$ integer (see, e.g., [16]). More generally, when variable stepsizes are used, Lax stability requires that for each $t>0$ the products $r\left(\Delta t_{k} A\right) \cdots r\left(\Delta t_{1} A\right), t=\Delta t_{1}+\cdots+\Delta t_{k}>0$, are bounded as the grid is refined (see, e.g., [17]). Moreover, if the semigroup $e^{t A}$ is bounded, i.e., $\left\|e^{t A}\right\| \leq C, t \geq 0$, then the stronger property of the collective boundedness of $r\left(\Delta t_{k} A\right) \cdots r\left(\Delta t_{1} A\right), t=\Delta t_{1}+\cdots+\Delta t_{k}>0$, independently of $t>0$ and the stepsizes $\Delta t_{i}$, is also of interest; i.e., one tries to ascertain

Received by the editor March 4, 1992 and, in revised form, August 7, 1992 and October 30, 1992.

1991 Mathematics Subject Classification. Primary 65J10, 65M12.

Key words and phrases. Holomorphic semigroups, sectorial operators, Banach spaces, rational approximation, strongly $\mathrm{A}(\theta)$-acceptable, stability, variable stepsizes.

(C) 1994 American Mathematical Society $0025-5718 / 94 \$ 1.00+\$ .25$ per page 
whether a constant $C^{\prime \prime}>0$ exists such that

$$
\left\|r\left(h_{K} A\right) \cdots r\left(h_{1} A\right)\right\| \leq C^{\prime}
$$

holds, for each finite sequence of positive numbers $h_{1}, \ldots, h_{k}$. Our goal is to establish the bound $(2$ ) for some of these rational methods.

Let us mention some results which are directly related to the present paper. The reader is also referred to [3] and [13] for recent overviews on this matter. Recall that a rational function $r(z)$ is called $A(\theta)$-acceptable $(0<\theta \leq \pi / 2)$ when it possesses no poles in the sector

$$
S_{\theta}=\{0\} \cup\left\{z \in C^{\prime}: z \neq 0,|\arg (-z)| \leq \theta\right\}
$$

and satisfies $|r(z)| \leq 1$ there. If, in addition, $|r(x)|<1$, then $r(z)$ is called strongly $A(\theta)$-acceptable. When $\theta=\pi / 2$, then the term $A$-acceptable is used. For example, the rational function associated with the backward Euler method is strongly A-acceptable, while the rational function associated with the CrankNicolson method is A-acceptable.

We allow now the semigroup $e^{l A}$ to be merely strongly continuous, i.e., not necessarily holomorphic, and also suppose that it is bounded. Given an Aacceptable rational function $r(z)$, the question arises as to whether the existence of the bound (2) is guaranteed. The answer is affirmative for dissipative generators in Hilbert spaces (see [12] and [20]). In [7] it was conjectured that, for a general Banach space $X$, there exists a constant $C=C^{\prime}(A, r)$ such that

$$
\left\|r(\Delta t A)^{k}\right\| \leq C k^{1 / 2} \quad(k \text { integer, } k \geq 1) \text {. }
$$

This conjecture was proved by Brenner and Thomée in [1]. Moreover, there is an example (cited in [1] and fully developed in [3]) where the growth in (3) is attained. Thus, it turns out that (2) cannot hold in general if $r$ is A-acceptable and $e^{t A}$ bounded and strongly continuous.

There are several improvements of (3) for sectorial operators $A$. Sectorial means (see, e.g., [6]) that for some $\theta \in(0, \pi)$ the spectrum of $A$ is contained in the sector $S_{y}$ and for a suitable constant $M \geq 1$ we have the bound

$$
\left\|(z-A)^{-1}\right\| \leq \frac{M}{|z|} \quad\left(z \in C, \quad z \notin S_{(g)}\right) .
$$

The set formed by all the linear, densely defined closed operators $A: D(A) \subset$ $X \rightarrow X$ satisfying condition (4) is denoted by $S(X, M, \theta)$. It is known (see, e.g., [15] and [22]) that $A$ is the generator of a bounded holomorphic semigroup if, and only if, $A$ belongs to a class $S(X, M, \theta)$, with $0<\theta<\pi / 2, M \geq 1$. Let $A \in S(X, M, \theta), 0<\theta<\pi / 2$. In [10] the convergence of rational strongly $\mathrm{A}(\theta)$-acceptable methods for bad initial data $u_{0} \in X$ is studied, in cases where $X$ is a Hilbert space and $A$ satisfies the more restrictive condition $\langle A x, x\rangle \in S_{f f}, x \in X$. Some of the ideas developed in [10] can also be applied to the study of the stability of rational methods in Banach spaces. For general Banach spaces, it was announced in [1] (see also [9] and [11]) that for any strongly $\mathrm{A}(\theta)$-acceptable rational function $r(z)$ there is a constant $C=C(r, \theta)$ such that

$$
\left\|r(\Delta t A)^{k}\right\|=M C: \quad(k \text { integer, } k \geq 1) ;
$$

i.e., the rational method based on $r(z)$ is stable for constant stepsizes. This result has been extended recently in [3] and [13], where (5) has been established 
for $\mathrm{A}(\theta)$-acceptable rational functions, i.e., allowing $|r(x)|=1$. (In [13] only A-acceptability is considered.) The results in [3] and [13] show the stability of the Crank-Nicolson scheme when applied, with constant time-steps, to parabolic problems in the maximum norm. In [13] variable stepsizes are also allowed, but the ratio between them must remain bounded (quasi-uniform mesh).

In this paper we give another improvement of (3). As in [13], we consider sectorial operators and variable stepsizes. However, we now assume strong $\mathrm{A}(\theta)$-acceptability rather than $\mathrm{A}(\theta)$-acceptability. As a result, stability can be shown for arbitrary sequences of stepsizes. The main contribution of the paper is the following theorem.

Theorem 1. Let $\theta \in(0, \pi / 2), M \geq 1$, and let $r(z)$ be a strongly $\mathrm{A}(\theta)$ acceptable rational function $r(z)$, consistent with the exponential, i.e., with $r(0)=r^{\prime}(0)=1$. Then there exists a constant $C=C(r, M, \theta)>0$ such that the bound

$$
\left\|r\left(h_{k} A\right) r\left(h_{k-1} A\right) \cdots r\left(h_{1} A\right)\right\| \leq C
$$

holds, for each complex Banach space $X$, each sectorial operator $A \in S(X, M, \theta)$, and any finite sequence $h_{1}, h_{2}, \ldots, h_{k}$ of positive numbers.

On the other hand, suppose that an $\mathrm{A}(\theta)$-acceptable rational function $r(z)$ is consistent with the exponential of order $p \geq 1$, i.e., $r(z)-e^{z}=O\left(z^{p+1}\right)$, $z \rightarrow 0$, and we maintain the remaining assumptions of Theorem 1 . Then, as pointed out in [8], we have the following error bound:

$$
\left\|e^{t A} u-\prod_{j=1}^{k} r\left(h_{j} A\right) u\right\| \leq C h^{q}\left\|A^{q} u\right\| \quad\left(u \in D\left(A^{q}\right), \quad 1 \leq q \leq p\right),
$$

where $t=h_{1}+\cdots+h_{k}$ and $h=\max \left\{h_{1}, \ldots, h_{k}\right\}$. This bound is easily proved by means of an argument similar to the one used in Theorem 4.4 in [9]. Here, the case $q=0$ is excluded and the stability cannot be obtained as a consequence of (7). However, the bound (7) gives the consistency of the method based upon the rational function and, along with Theorem 1 (see, e.g., [17], [18]), yields also convergence (without order), i.e., we have

$$
\lim \prod_{j=1}^{k} r\left(h_{j} A\right) u=e^{t A} u \quad(u \in X, t>0)
$$

where $h_{1}+\cdots+h_{k}=t$ and $\max \left\{h_{1}, \ldots, h_{k}\right\} \rightarrow 0^{+}$.

The proof of Theorem 1 relies on a new general bound for the operator norm of $f(A)$. Here, $A$ is again in $S(X, M, \theta), 0<\theta<\pi / 2$, and $f$ is any holomorphic, not necessarily rational, function defined in a neighborhood of the compact set $\{x\} \cup S_{\vartheta}$ on the Riemann sphere. Recall that the bounded operator $f(A): X \rightarrow X$ is then defined by means of the so-called Dunford-Taylor integral (see, e.g., [5])

$$
f(A)=f(x)+\frac{1}{2 \pi i} \int_{\Gamma}(f(z)-f(x))(z-A)^{-1} d z
$$

where $\Gamma$ is an appropriate path surrounding the spectrum of $A$ on the Riemann sphere. When $f(z)$ is a rational function or the exponential, the formula (8) is 
compatible with other available definitions of $f(A)$. The bound we are referring to is

$$
\|f(A)\| \leq 2 M\left\{3+\ln ^{+}\left(N_{\theta}(f) /\|f\|_{\theta}\right)\right\}\|f\|_{\theta},
$$

where $N_{\theta}$ is a certain functional, $\|f\|_{\theta}$ denotes the supremum of $|f(z)|$ in $S_{\theta}$, and $\ln ^{+}$stands for the positive part of the logarithm, $\ln ^{+} r=\max \{0, \ln r\}$.

In $\S 2$ we define the functional $N_{\theta}$ and establish (9). Section 3 is devoted to the proof of Theorem 1 and to a brief discussion of examples of its applicability.

\section{BOUNDS FOR GENERAL HOLOMORPHIC FUNCTIONS}

Here and later we will maintain the following notation. $H_{\theta}$ is the set of all holomorphic functions $f$ which we defined in an open neighborhood $\Omega_{f}$ of $\{\infty\} \cup S_{\theta}$ on the Riemann sphere. For $f \in H_{\theta}$, we will denote

$$
\|f\|_{\theta}=\sup \left\{|f(z)|: z \in S_{\theta}\right\}
$$

as well as

$$
\begin{aligned}
Z_{\theta}(f) & =\sup \left\{|f(z)-f(0)| /|z|: z \in S_{\theta}\right\}, \\
I_{\theta}(f) & =\sup \left\{|f(z)-f(\infty)||z|: z \in S_{\theta}\right\}, \\
K_{\theta}(f) & =\sqrt{\left(|f(0)|+|f(\infty)|+Z_{\theta}(f)\right)\left(|f(0)|+|f(\infty)|+I_{\theta}(f)\right)}
\end{aligned}
$$

and

$$
N_{\theta}(f)=|f(0)|+|f(\infty)|+\sqrt{Z_{\theta}(f) I_{\theta}(f)} .
$$

Let $f \in H_{\theta}$ and $\lambda>0$, and set $f_{\lambda}(z)=f(\lambda z), \lambda z \in \Omega_{f}$. Note that

$$
Z_{\theta}\left(f_{\lambda}\right)=\lambda Z_{\theta}(f) \text { and } I_{\theta}\left(f_{\lambda}\right)=\lambda^{-1} I_{\theta}(f),
$$

so that the quantity $N_{\theta}(f)$ is invariant against rescaling of $z$. In fact, as is easily checked, we have

$$
N_{\theta}(f)=\inf \left\{K_{\theta}\left(f_{\lambda}\right): \lambda>0\right\} .
$$

$N_{\theta}$ is also invariant against inversion $z \rightarrow z^{-1}$, which is another obvious symmetry of $S_{\theta}$.

Lemma 1. Let $\theta \in(0, \pi / 2)$ and $M \geq 1$. The bound

$$
\|f(A)\| \leq \begin{cases}(2 M / \pi) N_{\theta}(f) & \text { if } N_{\theta}(f) \leq\|f\|_{\theta}, \\ (2 M / \pi)\left\{1+\ln \left(N_{\theta}(f) /\|f\|_{\theta}\right)\right\}\|f\|_{\theta} & \text { if } N_{\theta}(f) \geq\|f\|_{\theta}\end{cases}
$$

holds for each Banach space $X$, each operator $A$ in $S(X, M, \theta)$, and each holomorphic function $f \in H_{\theta}$ with $f(0)=f(\infty)=0$.

Proof. Observe that both sides in (12) are homogeneous in $f \in H_{\theta}$, so that there is no loss of generality in assuming that $\|f\|_{\theta}=1$. As $f(\infty)=0$, the operator $f(A)$ is given by the Dunford-Taylor integral (see (8))

$$
f(A)=\frac{1}{2 \pi i} \int_{\Gamma} f(z)(z-A)^{-1} d z
$$

where $\Gamma$ is a path in the complex plane that leaves the sector $S_{\theta}$ to its left. In fact, since $f(0)=0$, it is straightforward to verify that $\Gamma$ can be chosen as the positive boundary of $S_{\theta}$. Set, for each positive $r$,

$$
\omega(f, r, \theta)=\max \left(\left|f\left(r e^{i \theta}\right)\right|,\left|f\left(r e^{-i \theta}\right)\right|\right)
$$


and take norms in (13). The result is

$$
\|f(A)\| \leq \frac{M}{\pi} \int_{0}^{+\infty} \omega(f, r, \theta) r^{-1} d r .
$$

Recall now the definitions $Z_{\theta}(f)$ and $I_{\theta}(f)$. It is clear that the inequalities

$$
\omega(f, r, \theta) \leq\left\{\begin{array}{l}
r Z_{\theta}(f) \\
\|f\|_{\theta}=1 \\
I_{\theta}(f) / r
\end{array}\right.
$$

are valid for $r>0$. Hence, for $0<a \leq b<+\infty$, we can transform (14) into

$$
\begin{aligned}
\|f(A)\| & \leq \frac{M}{\pi}\left\{\int_{0}^{a} Z_{\theta}(f) d r+\int_{a}^{b} r^{-1} d r+\int_{b}^{+\infty} I_{\theta}(f) r^{-2} d r\right\} \\
& \left.=(M / \pi)\left\{a Z_{\theta}(f)+\ln (b / a)+I_{\theta}(f) / b\right)\right\} .
\end{aligned}
$$

If $N_{\theta}(f)=\sqrt{Z_{\theta}(f) I_{\theta}(f)} \leq 1$, we take

$$
a=b=\sqrt{I_{\theta}(f) Z_{\theta}(f)^{-1}}
$$

in (15), whereas, if $N_{\theta}(f)>1$, we choose

$$
a=Z_{\theta}(f)^{-1} \leq I_{\theta}(f)=b .
$$

This proves (12).

Theorem 2. Let $\theta \in(0, \pi / 2)$ and $M \geq 1$. The bound

$$
\|f(A)\| \leq 2 M\left\{3+\ln ^{+}\left(N_{\theta}(f) /\|f\|_{\theta}\right)\right\}\|f\|_{\theta}
$$

holds for each Banach space $X$, each operator $A$ in $S(X, M, \theta)$, and each holomorphic function $f \in H_{\theta}$.

Proof. We begin by proving the slightly different bound

$$
\|f(A)\| \leq 2 M\left\{3+\ln ^{+}\left(K_{\theta}(f) /\|f\|_{\theta}\right)\right\}\|f\|_{\theta} .
$$

We can assume again that $\|f\|_{\theta}=1$. Define the new complex function $g \in H_{\theta}$ by means of

$$
g(z)=f(z)-f(0)(1-z)^{-1}+f(\infty) z(1-z)^{-1},
$$

so that $g(\infty)=g(0)=0$. On the other hand, given that $A \in S(X, M, \theta)$, we have

$$
\left\|(1-A)^{-1}\right\| \leq M
$$

and

$$
\begin{aligned}
\left\|A(1-A)^{-1}\right\| & =\left\|(A-1+1)(1-A)^{-1}\right\| \\
& \leq\left\|(1-A)^{-1}\right\|+1 \leq 1+M \leq 2 M
\end{aligned}
$$

so that

$$
\begin{aligned}
\|f(A)\| & =\left\|g(A)+f(0)(1-A)^{-1}+f(\infty) A(1-A)^{-1}\right\| \\
& \leq\|g(A)\|+|f(0)|\left\|(1-A)^{-1}\right\|+|f(\infty)|\left\|A(1-A)^{-1}\right\| \\
& \leq\|g(A)\|+3 M .
\end{aligned}
$$


Therefore, in order to prove (17), it suffices to establish that

$$
\|g(A)\| \leq 2 M\left\{3 / 2+\ln ^{+} K_{\theta}(f)\right\} .
$$

If $z$ belongs to $S(\theta)$, then $\left|(1-z)^{-1}\right| \leq 1$ and $\left|z(1-z)^{-1}\right| \leq 1$, because $\mathfrak{R}(z) \leq 0$. Consequently,

$$
\|g\|_{\theta} \leq\|f\|_{\theta}+|f(0)|+|f(\infty)| \leq 3 .
$$

Now fix $z \neq 0$ in $S_{\theta}$. We have

$$
\begin{aligned}
|g(z) / z| & \leq|f(z)-f(0)| /|z|+\left|f(0) z^{-1}-f(0) z^{-1}(1-z)^{-1}\right|+|f(\infty)| /|1-z| \\
& \leq|f(z)-f(0)| /|z|+|f(0)| /|1-z|+|f(\infty)| /|1-z| \\
& \leq|f(0)|+|f(\infty)|+Z_{\theta}(f),
\end{aligned}
$$

and taking the supremum in $z \in S_{\theta}$ in the above inequality, we conclude that

$$
Z_{\theta}(g) \leq|f(0)|+|f(\infty)|+Z_{\theta}(f) .
$$

In the same way we prove that

$$
I_{\theta}(g) \leq|f(0)|+|f(\infty)|+I_{\theta}(f),
$$

and combining the last two inequalities, we deduce

$$
N_{\theta}(g)=\sqrt{Z_{\theta}(g) I_{\theta}(g)} \leq K_{\theta}(f) .
$$

We now apply Lemma 1 to $g$. Suppose first that $N_{\theta}(g) \leq\|g\|_{\theta}$. In this case, by virtue of (19),

$$
\|g(A)\| \leq(2 M / \pi) N_{\theta}(g) \leq 3 M
$$

and (18) holds. Suppose now that $N_{\theta}(g) \geq\|g\|_{\theta}$. By (20) we can write

$$
\begin{aligned}
\|g(A)\| & \leq(2 M / \pi)\left\{1+\ln \left(N_{\theta}(g) /\|g\|_{\theta}\right)\right\}\|g\|_{\theta} \\
& \leq(2 M / \pi)\left(1+\ln K_{\theta}(f)-\ln \|g\|_{\theta}\right)\|g\|_{\theta},
\end{aligned}
$$

and since

$$
-\sigma \ln \sigma \leq e^{-1} \quad(\sigma>0),
$$

and by (19), we arrive at

$$
\begin{aligned}
\|g(A)\| & \leq \frac{2 M}{\pi} 3\left(1+\ln K_{\theta}(f)\right)+2 M /(e \pi) \\
& \leq 2 M\left(1+\ln K_{\theta}(f)\right)+M
\end{aligned}
$$

which implies (18).

It remains to prove (16) from (17). Let us fix $\lambda>0$ and denote $f_{\lambda}(z)=$ $f(\lambda z), \lambda z \in \Omega_{f}$. The operator $\lambda^{-1} A$ belongs again to $S(X, M, \theta)$, thus the bound (17) gives

$$
\|f(A)\|=\left\|f_{\lambda}\left(\lambda^{-1} A\right)\right\| \leq 2 M\left\{3+\ln ^{+}\left(\frac{K_{\theta}\left(f_{\lambda}\right)}{\|f\|_{\theta}}\right)\right\}\|f\|_{\theta},
$$

and (16) now follows easily after taking the infimum with respect to $\lambda>0$ and recalling (11).

\section{RATIONAL APPROXIMATIONS WITH VARIABLE TIME-STEPS}

We begin this section with the proof of Theorem 1. First, two simplifications are made. 
It is known that for each pair of constants $M \geq 1$ and $0<\theta<\pi / 2$, there exists a new pair $M^{\prime} \geq 1$ and $0<\theta^{\prime}<\theta$ such that $S(X, M, \theta) \subset$ $S\left(X, M^{\prime}, \theta^{\prime}\right)$ (see, e.g., [6]). It is clear, by virtue of the maximum modulus principle, that

$$
|r(z)|<1 \quad\left(z \in S_{\theta^{\prime}}, \quad z \neq 0\right) .
$$

For $A \in S(X, M, \theta)$ we could argue with $A$ as an element in $S\left(X, M^{\prime}, \theta^{\prime}\right)$; thus there is no loss of generality if we assume that

$$
|r(z)|<1 \quad\left(z \in S_{\theta}, \quad z \neq 0\right) .
$$

Second, fix $A \in S(X, M, \theta)$. It is well known (see [15]) that $A$ generates a holomorphic semigroup $\exp (t A), t \geq 0$, and that for some constant $L(\theta)>0$ we have

$$
\|\exp (t A)\| \leq L(\theta) M \quad(t \geq 0) .
$$

Denote by $\|\cdot\|$ the norm in $X$, and then define a new norm $\|\cdot\|^{*}$ in $X$ by means of

$$
\|x\|^{*}=\sup \{\|\exp (t A) x\|: t \geq 0\} \quad(x \in X) .
$$

Observe that

$$
\|x\| \leq\|x\|^{*} \leq L(\theta) M\|x\| \quad(x \in X)
$$

and that, with respect to the new norm $\|\cdot\|^{*}$, the semigroup $\exp (t A), t \geq 0$, becomes a semigroup of contractions. Thus, after changing the norm in $X$, if necessary, there is no loss of generality if we assume in the rest of the proof that the $\exp (t A), t \geq 0$, is a semigroup of contractions on $X$ or, equivalently because of the Hille-Yosida theorem (see, e.g., [15]), that

$$
\left\|(1-h A)^{-1}\right\| \leq 1 \quad(h>0) .
$$

After the above simplifications we begin the proof. For each integer $k \geq 1$ we define

$$
C_{k}=\sup \left\|r\left(\lambda_{k} A\right) r\left(\lambda_{k-1} A\right) \cdots r\left(\lambda_{1} A\right)\right\|,
$$

where $\lambda_{1}, \ldots, \lambda_{k}$ range through all the finite sequences of length $k$, formed with positive real numbers. Since the composition appearing in (24) is commutative, and since we may replace the operator $A$ by $\lambda_{1} A$, which is also in $S(X, M, \theta)$, we see that it is sufficient to consider monotone sequences $\lambda_{k} \geq \lambda_{k-1} \geq \cdots \geq \lambda_{2} \geq \lambda_{1}$ with $\lambda_{1}=1$.

Suppose we have proved the following fact: There exists a sequence $\left\{\varepsilon_{k}\right\}_{k=1}^{+\infty}$ of positive numbers, depending only on the rational function $r(z)$, such that

$$
\sum_{k=1}^{+\infty} \varepsilon_{k}<+\infty
$$

and such that, for each integer $k \geq 2$ and any monotone sequence $\lambda_{k} \geq \cdots \geq \lambda_{1}$ with $\lambda_{1}=1$, the bound

$$
\left\|r\left(\lambda_{k} A\right) r\left(\lambda_{k-1} A\right) \cdots r\left(\lambda_{1} A\right)\right\| \leq \varepsilon_{k}+C_{k-1}
$$

holds. Then

$$
C_{k} \leq \varepsilon_{k}+C_{k-1} \quad(k \geq 2),
$$


which implies

$$
C_{k} \leq C_{1}+\sum_{m=2}^{k} \varepsilon_{m} \quad(k \geq 2),
$$

and the proof is finished, because of (25).

Now we are going to construct a sequence $\left\{\varepsilon_{k}\right\}_{k=1}^{+\infty}$ satisfying (25) and (26). Since the rational function $r(z)$ is strongly $\mathrm{A}(\theta)$-acceptable and satisfies (22), it is easy to select a constant $c>0$ such that

$$
|r(z)| \leq \begin{cases}e^{-c|z|} & \text { if }|z| \leq 1, \quad z \in S_{\theta}, \\ e^{-c} & \text { if }|z| \geq 1, \quad z \in S_{\theta} .\end{cases}
$$

Remember that $r(z)$ is also consistent with the exponential, i.e.,

$$
r(0)=r^{\prime}(0)=1 \text {, }
$$

and hence there exists $L \geq 2$ with

$$
\left|r(z)-(1-z)^{-1}\right| \leq \begin{cases}L|z|^{2} & \text { if } z \in S_{\theta},|z| \leq 1 \\ L & \text { if } z \in S_{\theta},|z| \geq 1\end{cases}
$$

Finally, we take a number $\alpha \in(1 / 2,1)$, and then choose a constant $L^{*} \geq L$ such that

$$
L e^{c k^{-\alpha}} e^{-c k^{(1-\alpha)}} \leq L^{*} k^{-2 \alpha} \quad(k \geq 1) .
$$

We will set, for each integer $k \geq 1$,

$$
\varepsilon_{k}^{*}=L^{*} k^{-2 \alpha}
$$

and

$$
\varepsilon_{k}=\sup \left\{2 M\left\{3+\ln ^{+}\left(N^{*} / x\right)\right\} x: 0<x \leq \varepsilon_{k}^{*}\right\}
$$

where

$$
N^{*}:=1+\sqrt{\left(1+Z_{\theta}(r)\right)\left(1+I_{\theta}(r)\right)(1-|r(\infty)|)^{-1}} \geq 1 .
$$

Let us check that (25) holds. Observe that for small enough $x>0$ the expression $x \ln ^{+}\left(N^{*} / x\right)$ is increasing and hence, for a large enough integer $k$, we have

$$
\varepsilon_{k}=2 M\left\{3+\ln ^{+}\left(N^{*} / \varepsilon_{k}^{*}\right)\right\} \varepsilon_{k}^{*} \leq 2 M\left\{3+\ln N^{*}\right\} \varepsilon_{k}^{*}+2 M\left|\ln \varepsilon_{k}^{*}\right| \varepsilon_{k}^{*},
$$

and (25) follows since both series $\sum_{k=1}^{\infty} \varepsilon_{k}^{*}=\sum_{k=1}^{\infty} L^{*} k^{-2 \alpha}$ and $\sum_{k=1}^{\infty}\left|\ln \varepsilon_{k}^{*}\right| \varepsilon_{k}^{*}$ $=\sum_{k=1}^{\infty} 2 L^{*} \ln L^{*} \alpha k^{-2 \alpha} \ln k$ are convergent because of $\alpha>1 / 2$.

Now we are going to establish (26). Let $\lambda_{k} \geq \cdots \geq \lambda_{1}$ be a monotone sequence of length $k \geq 2$, with $\lambda_{1}=1$. Denote

$$
g(z)=r\left(\lambda_{k} z\right) \cdots r\left(\lambda_{2} z\right)
$$

and

$$
f(z)=g(z)\left(r(z)-(1-z)^{-1}\right) .
$$

We can write

$$
r\left(\lambda_{k} A\right) \cdots r\left(\lambda_{2} A\right) r(A)=f(A)+g(A)(1-A)^{-1},
$$

so that, because of assumption (23) and definition (24),

$$
\left\|r\left(\lambda_{k} A\right) \cdots r\left(\lambda_{2} A\right) r(A)\right\| \leq\|f(A)\|+C_{k-1},
$$

and what we need is to estimate $\|f(A)\|$. 
Set

$$
f_{m}(z)=r\left(\lambda_{m} z\right) \quad(2 \leq m \leq k)
$$

and

$$
f_{1}(z)=r(z)-(1-z)^{-1}
$$

For $z \in S_{\theta}$ we have

$$
z(f(z)-f(\infty))=z \sum_{m=1}^{k} f_{k}(z) \cdots f_{m+1}(z)\left(f_{m}(z)-r(\infty)\right) r(\infty)^{m-1}
$$

and therefore, taking (10) into account,

$$
\begin{aligned}
|z(f(z)-f(\infty))| & \leq \sum_{m=1}^{k} I_{\theta}\left(f_{m}\right) \mu^{m-1} \\
& =I_{\theta}\left(f_{1}\right)+\sum_{m=2}^{k} I_{\theta}(r) \lambda_{m}^{-1} \mu^{m-1},
\end{aligned}
$$

where $\mu=|r(\infty)|$. Hence, because $I_{\theta}\left(f_{1}\right) \leq 1+I_{\theta}(r)$ and $\lambda_{m} \geq 1$, we find that

$$
I_{\theta}(f) \leq\left(1+I_{\theta}(r)\right)(1-\mu)^{-1} \text {. }
$$

For $z \in S_{\theta}, z \neq 0$, we have also

$$
\begin{aligned}
\left|(f(z)-f(0)) z^{-1}\right| & =\left|f(z) z^{-1}\right| \leq\left|z^{-1}\left(r(z)-(1-z)^{-1}\right)\right| \\
& \leq\left|(r(z)-1) z^{-1}\right|+\left|\left((1-z)^{-1}-1\right) z^{-1}\right| \leq Z_{\theta}(r)+1 ;
\end{aligned}
$$

thus

$$
Z_{\theta}(f) \leq 1+Z_{\theta}(r)
$$

which together with (32) and (33) yields

$$
N_{\theta}(f) \leq \mu^{k}+\sqrt{\left(1+Z_{\theta}(r)\right)\left(1+I_{\theta}(r)\right)(1-\mu)^{-1}} \leq N^{*} .
$$

According to Theorem 2, we find

$$
\|f(A)\| \leq 2 M\left\{3+\ln ^{+}\left(N_{\theta}(f) /\|f\|_{\theta}\right)\right\}\|f\|_{\theta}
$$

and, because of the definition (31), the proof of Theorem 1 will be completed once we establish

$$
\|f\|_{\theta} \leq \varepsilon_{k}^{*}
$$

Let $z \in S_{\theta}$. If $|z| \leq k^{-\alpha}$, then, by (28), we have

$$
|f(z)| \leq\left|f_{1}(z)\right|=\left|r(z)-(1-z)^{-1}\right| \leq L k^{-2 \alpha} \leq \varepsilon_{k}^{*},
$$

while if $|z| \geq k^{-\alpha}$, then, by (27), (28), and (29), we have

$$
\begin{aligned}
|f(z)| & \leq L\left|r\left(\lambda_{k} z\right) \cdots r\left(\lambda_{2} z\right)\right| \leq L\left(e^{-c k^{-\alpha}}\right)^{k-1} \\
& \leq L e^{c k^{-\alpha}} e^{-c k^{(1-\alpha)}} \leq L^{*} k^{-2 \alpha}=\varepsilon_{k}^{*}
\end{aligned}
$$

so that in either case $|f(z)| \leq \varepsilon_{k}^{*}, z \in S_{\theta}$, and (35) is satisfied.

We end the paper with a brief discussion about further extensions of Theorem 1. First we consider the case where $A$ generates a holomorphic semigroup but this semigroup is not bounded, so that for some $\omega>0$ we have 
$(A-\omega) \in S(X, M, \theta)$. This case occurs for example in parabolic equations with a source term, or in parabolic systems of PDEs. The same argument used in the final section in [13] shows that for any consistent, strongly $\mathrm{A}(\theta)$-acceptable rational function $r(z)$, there exist two constants $C=C(M, \theta, r)>0$ and $J=J(M, \theta, r)>0$ such that

$$
\left\|r\left(h_{k} A\right) \cdots r\left(h_{1} A\right)\right\| \leq C e^{J \omega t} \quad\left(t=h_{1}+\cdots+h_{k}>0\right) .
$$

On the other hand, when discretization in space is considered as well, it is necessary to analyze the stability of a family of problems

$$
\left\{\begin{array}{l}
u_{\Delta x}^{\prime}(t)=A_{\Delta x} u_{\Delta x}(t), \\
u_{\Delta x}(0)=u_{\Delta x, 0}
\end{array}\right.
$$

which depends on a parameter $\Delta x \rightarrow 0+$ (see, e.g., [14] and [18]). If $A_{\Delta x} \in$ $S\left(X_{\Delta x}, M_{\Delta x}, \theta\right)$, then the stability will be guaranteed as long as the constants $M_{\Delta x}$ remain bounded when $\Delta x \rightarrow 0+$, a condition that of course many numerical procedures fulfill (see [2] and [21]).

Finally, Theorem 1 could be useful in the theory of discretizations of ODEs, when deriving stability bounds which are independent of the stiffness of the problem (see [4] and [19]).

\section{ACKNOWLEDGMENT}

The author is most grateful to Professor Sanz-Serna for reading the manuscript and to Professor Larsson for several valuable suggestions.

\section{BIBLIOGRAPHY}

1. P. Brenner and V. Thomée, On rational approximations of semigroups, SIAM J. Numer. Anal. 16 (1979), 683-694.

2. P. Brenner, V. Thomée, and L. B. Wahlbin, Besov spaces and applications to difference methods for initial value problems, Lecture Notes in Math., vol. 434, Springer-Verlag, Berlin, 1975.

3. M. Crouzeix, S. Larsson, S. Piskarev, and V. Thomée, The stability of rational approximations of analytic semigroups, BIT 33 (1993), 74-84.

4. K. Dekker and J. G. Verwer, Stability of Runge-Kutta methods for stiff nonlinear differential equations, North-Holland, Amsterdam, 1984.

5. N. Dunford and L. Schwartz, Linear operators, part I, Interscience, New York, 1958.

6. H. O. Fattorini, The Cauchy problem, Addison-Wesley, Reading, MA, 1983.

7. R. Hersh and T. Kato, High-accuracy stable difference schemes for well-posed initial value problems, SIAM J. Numer. Anal. 16 (1979), 670-682.

8. S. Larsson, private communication.

9. S. Larsson, V. Thomée, and L. B. Wahlbin, Finite-element methods for a strongly damped wave equation, IMA J. Numer. Anal. 11 (1991), 115-142.

10. M. N. LeRoux, Semidiscretizations in time for parabolic problems, Math. Comp. 33 (1979), 919-931.

11. C. Lubich and O. Nevanlinna, On resolvent conditions and stability estimates, BIT 31 (1991), 293-313.

12. R. MacKelvey, Spectral measures, generalized resolvents, and functions of positive type, J. Math. Anal. Appl. 11 (1965), 447-477.

13. C. Palencia, A stability result for sectorial operators in Banach spaces, SIAM J. Numer. Anal. 30 (1993), 1373-1384. 
14. C. Palencia and J. M. Sanz-Serna, An extension of the Lax-Richtmyer theory, Numer. Math. 44 (1984), 109-115.

15. A. Pazy, Semigroups of linear operators and applications to partial differential equations, Springer-Verlag, New York, 1983.

16. R. D. Richtmyer and K. W. Morton, Difference methods for initial value problems, 2nd ed., Interscience, New York, 1967.

17. J. M. Sanz-Serna, Stability and convergence in numerical analysis. 1: Linear problems-a simple comprehensive account, Nonlinear Equations and Applications (J. K. Hale and P. Martínez Amores, eds.), Pitman, Boston, 1985, pp. 64-113.

18. J. M. Sanz-Serna and C. Palencia, A general equivalence theorem in the theory of discretization methods, Math. Comp. 45 (1985), 143-152.

19. J. M. Sanz-Serna and J. G. Verwer, Stability and convergence at the PDE/stiff ODE interface, Appl. Numer. Math. 5 (1989), 117-132.

20. B. Sz.-Nagy and C. Foias, Harmonic analysis of operators on Hilbert spaces, North-Holland, Amsterdam, 1970.

21. V. Thomée, Finite difference methods for linear parabolic equations, Handbook for Numerical Analysis, vol. I. (P. G. Ciarlet and J. L. Lions, eds.), North-Holland, Amsterdam, 1990.

22. K. Yosida, Functional analysis, 6th ed., Springer-Verlag, Berlin, 1980.

Departamento de Matemática Aplicada y Computación, Facultad de Ciencias, UniVersidad de Valladolid, Valladolid, Spain

E-mail address: palencia@cpd.uva.es 